\title{
Rabaska
}

Revue d'ethnologie de l'Amérique française

MOFFET, ClAUdE. L'Union sardinière du St-Laurent à Saint-André-de-Kamouraska - 1891-1898. Québec, Éditions GID, 2018, 162 p. ISBN 978-2-89634-383-6

\section{René Gagnon}

Volume 17, 2019

URI : https://id.erudit.org/iderudit/1066037ar

DOI : https://doi.org/10.7202/1066037ar

Aller au sommaire du numéro

Éditeur(s)

Société québécoise d'ethnologie

ISSN

1703-7433 (imprimé)

1916-7350 (numérique)

Découvrir la revue

Citer ce compte rendu

Gagnon, R. (2019). Compte rendu de [MOFFET, CLAUDE. L'Union sardinière du St-Laurent à Saint-André-de-Kamouraska - 1891-1898. Québec, Éditions GID,

2018, 162 p. ISBN 978-2-89634-383-6]. Rabaska, 17, 332-335.

https://doi.org/10.7202/1066037ar d'utilisation que vous pouvez consulter en ligne.

https://apropos.erudit.org/fr/usagers/politique-dutilisation/ 
à la fin de son ouvrage. Quant à l'éditeur, il ne semble pas avoir bien fait son travail, car un certain nombre de fautes d'orthographe ou de grammaire déparent le recueil et dérangent la lecture...

Mais la formule est tout de même de mise : «À votre santé ! »... «Entonne dans ta gorge / De ce bon cidre nouviau ; / Car j'entends nos pressoirs / Qui crient miséricorde / Qui disent toute l'année / Qu'on en boira core » (p. 42). Oui, « À la première auberge nous boirons (bis) [...] Là nous boirons si les filles sont belles [...] Là nous boirons si le vin est bon » (p. 164).

Aurélien Boivin

Littérature, Université Laval

Moffet, Claude. L'Union sardinière du St-Laurent à Saint-André-deKamouraska - 1891-1898. Québec, Éditions GID, 2018, 162 p. ISBN 9782-89634-383-6.

Au détour de recherches généalogiques sur ses origines familiales, Claude Moffet, l'auteur de cette monographie, se découvre un lien de parenté avec Jean-Baptiste-Eugène Letellier de Saint-Just, un marchand de Québec qui fonde et dirige l'Union sardinière du St-Laurent. Moffet publie d'abord à compte d'auteur la biographie du personnage en 2014. Puis, sur la base des données réunies à ce moment, il s'engage, en amateur d'histoire éclairé, dans la réalisation du présent ouvrage.

Il faut dire que le personnage de J.-B.-E. Letellier - tel qu'il abrégeait lui-même son nom et que l'auteur le désigne - a de quoi exciter la curiosité des chercheurs. Cependant, ce n'est pas d'abord du neveu du politicien Luc Letellier de Saint-Just dont il est question ici, mais bien d'une ambitieuse entreprise que le riche commerçant de gros et entrepreneur tente d'exploiter. Natif de Rivière-Ouelle, comme son oncle, Letellier apprend vite à exploiter le potentiel commercial des richesses issues du fleuve puisqu'il en fait la distribution et l'exportation. À la suite de quelques voyages en Europe, il découvre la saveur incomparable des sardines conservées dans l'huile d'olive vierge grâce au procédé d'appertisation, une méthode de conditionnement stérile inventée en 1795 et améliorée au cours des décennies suivantes. Or, les pêcheurs du Bas Saint-Laurent capturent de grandes quantités de sardines dans leurs engins de pêche, dont ils ne gardent qu'une partie infime pour le commerce ou pour la consommation, se débarrassant du reste. L'entrepreneur conçoit donc le projet de lancer une conserverie, de mettre en marché ce nouveau produit et d'en faire, comme en Europe, une marchandise de luxe. C'est à Saint-André-de-Kamouraska qu'il va implanter son usine, sur un terrain appartenant au puissant industriel et député provincial Charles-Alfred 
Desjardins, à proximité du quai nouvellement construit. En 1890-1891, selon l'hypothèse de l'auteur, Letellier fait ériger la conserverie et une partie des dépendances avec les matériaux probablement fournis par l'un des futurs actionnaires de l'Union sardinière, Joseph Honoré Gignac, de Québec, l'un des plus importants marchands de charpenterie, de bois d'œuvre et de boiserie de l'est de la province.

La production s'amorce en 1892 après l'embauche d'un directeur français spécialisé en la matière, Georges Bouillet, qui débarque en Amérique en mai. Un mois auparavant, Letellier avait signé un contrat d'approvisionnement avec une vingtaine de pêcheurs du village en vue d'obtenir la matière première suffisant à l'exploitation de la conserverie. En haute saison, entre 300 et 400 personnes vont y travailler au cours de cette première année. Pour loger ce personnel, Letellier a fait construire des bâtiments à proximité de l'usine : des logements pour les familles et des baraquements avec chambres individuelles pour les célibataires, un pour les femmes et un autre pour les hommes. Le démarrage de l'entreprise ne baigne cependant pas dans l'huile. D'une part, cette année-là, la sardine se fait attendre jusqu'en juillet. D'autre part, Letellier doit patienter un an avant de bénéficier d'une exemption de douanes sur l'importation de l'huile d'olive italienne, qu'il a sollicitée en avril (1892) auprès des autorités fédérales. D'allégeance publiquement libérale, l'industriel prête le flanc aux attaques partisanes : il s'ensuit une guerre de mots dans les journaux de l'époque. Tandis que L'Événement, d'obédience conservatrice, accuse Letellier de chercher à profiter de ses influences, L'Électeur, organe du parti libéral, défend les requêtes et prétentions de l'industriel. Letellier obtiendra finalement une remise de ses droits de douane en mai 1893. Dans l'intervalle, la saison 1892 aura servi de banc d'essai.

L'année 1893 s'amorce sous de meilleurs auspices. Dès le 16 janvier, Charles-Alfred Desjardins signe une promesse de vente des terrains occupés par l'Union sardinière à une éventuelle société par actions éponyme. Six mois plus tard, les parties concluent l'acte de vente. Toujours en janvier, le 19 cette fois, un acte d'incorporation est signé devant notaire, puis ratifié le 6 février. C'est dire que J.-B.-E. Letellier vient de partager le fardeau des investissements et des risques financiers avec quatorze autres actionnaires, la plupart appartenant à la bourgeoisie de Québec, de la Côte-du-Sud ou du Bas-Saint-Laurent, notables, commerçants, industriels, etc. Ainsi, l'avocat Louis-Philippe Chaloult de Québec - qui possède une résidence d'été à Kamouraska - devient le président d'un conseil d'administration qui procède derechef à l'embauche de J.-B.-E. Letellier comme gérant de l'entreprise. Dès le début mars, la sardine à l'huile produite l'année précédente est offerte dans plus d'une quarantaine d'épiceries à Québec. Pour autant, les adversaires politiques de Letellier ne baissent pas les bras. Ils accusent l'Union sardinière 
de détailler du petit hareng plutôt que de la sardine. La guerre de mots se poursuit par journaux interposés, Letellier et Chaloult devant rectifier les faits et protéger leur réputation. Et le marché de la sardine à l'huile, par la même occasion. Profitant des informations dont il dispose comme fournisseur de vin de messe à l'archevêché de Québec, Letellier frappe un grand coup en matière de marketing puisqu'il organise, à l'occasion du passage dans le Bas-SaintLaurent de l'archevêque de Québec, $\mathrm{M}^{\mathrm{gr}}$ Louis-Nazaire Bégin, une grande fête au village de Saint-André les 16 et 17 juillet dont le clou est la bénédiction de la conserverie, de ses dépendances et de son personnel. Letellier, en prospère commerçant de gros, connaît ses réseaux de distribution, si bien qu'en peu de temps, il réussit à mettre son produit en marché dans l'ensemble du Québec, voire à l'extérieur, comme en Ontario et en Nouvelle-Angleterre. Ses efforts s'appuient sur la publicité et l'information publiée dans les journaux, tant de langue anglaise que française. En septembre, l'Union sardinière remporte une médaille de l'Exposition universelle de Chicago pour la qualité de son produit. En décembre, l'Imperial Institute de Londres, mis sur pied par le Prince de Galles dans le but de promouvoir les meilleures denrées de l'empire britannique, invite la conserverie à commercialiser la sardine à l'huile de Saint-André sur le marché anglais où elle trouverait un « excellent» accueil.

Les activités de l'Union sardinière se poursuivent au cours des années subséquentes malgré un approvisionnement imprévisible. Parfois trop grosse, la sardine ne se laisse pas mettre en boîte : il faut alors la conserver en saumure, ce qui procure un produit bon marché et beaucoup moins rentable ; parfois trop rare, elle force la conserverie à écouler en bidons la coûteuse huile d'olive vierge inutilisée. Aussi, après six ans d'aléas divers, Letellier et ses associés abandonnent. En février 1899, ils rétrocèdent les installations de l'Union sardinière à C.-A. Desjardins qui les intégrera à ses autres établissements industriels.

L'historienne ou l'historien chevronné aura peut-être espéré trouver un traité sur l'Union sardinière ; il mettra plutôt la main sur le compte rendu d'une recherche menée en amateur. Dans sa « Note au lecteur », Moffet pose les balises de sa démarche. D'une part, il veut respecter la lettre plutôt que l'esprit des documents qu'il met au jour. Au fil des chapitres, l'ouvrage contient ainsi la reproduction intégrale des contrats, coupures de presse et autres articles consultés. D'autre part, l'auteur n'a utilisé que des ressources disponibles en ligne pour retracer l'histoire de l'entreprise. Ces choix imposent des contraintes strictes et engendrent des lacunes évidentes dans la quantité et la qualité de la documentation exploitée. L'auteur a toutefois l'honnêteté de communiquer son intention d'entrée de jeu. Vue sous cet angle, la publication de Claude Moffet projette un éclairage inédit, et bienvenu, sur l'histoire du commerce et de l'industrie québécoises de l'époque. Elle remet radicalement 
en question la croyance faussement et largement répandue que les Canadiens français n'avaient pas le sens des affaires ni l'esprit entrepreneurial. Elle ouvre la porte à des recherches sérieuses dans le domaine, elle invite à mieux documenter ces aspects négligés de notre histoire.

René Gagnon

Société d'histoire et de généalogie de la Côte-du-Sud

Paradis, Isabelle. Les Maisons de l'Île m'ont raconté... Atelier découverte pour petits et grands curieux. [Sainte-Famille, Île d'Orléans], Fondation François-Lamy, 2018, 24 p.

À l'heure où l'avenir du patrimoine bâti paraît plus sombre que jamais et suscite les plus vives inquiétudes devant le triste sort réservé à des icônes de l'architecture domestique et bourgeoise, comme à Lévis et Chambly de triste mémoire, et que l'idée s'ancre de plus en plus dans les esprits qu'un patrimoine qui s'appauvrit est un patrimoine qui dépérit avec notre identité, il est encourageant et réjouissant d'entendre des voix se lever et de voir des gestes posés pour changer les mentalités.

Isabelle Paradis, restauratrice spécialisée dans le traitement des œuvres en pierre au Centre de conservation du Québec (CCQ), est de cette classe. Passionnée par le patrimoine du Québec et celui de l'Île d'Orléans en particulier, elle a constitué une banque de données sur les maisons ancestrales au cours de ses travaux de restauration. Ces derniers l'ont amenée à approfondir ses recherches et à publier des articles précieux pour l'enrichissement de nos connaissances sur les maisons patrimoniales. Son expertise rayonne déjà auprès des architectes des quatre coins du Québec et on espère voir paraître bientôt son Guide sur la pierre et les mortiers, encore en chantier mais qui devrait bientôt enrichir une collection inaugurée par le CCQ avec la publication d'un premier guide technique sur Toit. Bois. Bardeau (Publications du Québec, 2016), devenu rapidement la référence par excellence pour les toitures de bois.

Entretemps, son analyse sur les finis architecturaux, parue dans Rabaska (vol. 16, 2018, p. 57-62), révélait déjà le caractère insoupçonné de l'étude des finis peints pour connaître plus en détail l'histoire des bâtiments anciens. «Encore mal connue en conservation architecturale au Québec, ce type d'étude, soutient la spécialiste, commence peu à peu à faire sa place dans les projets de restauration. " Autant « pour une maison historique, un bâtiment institutionnel ou une église, affirme-t-elle avec conviction, les couches stratigraphiques des finis peints permettent d'analyser les 